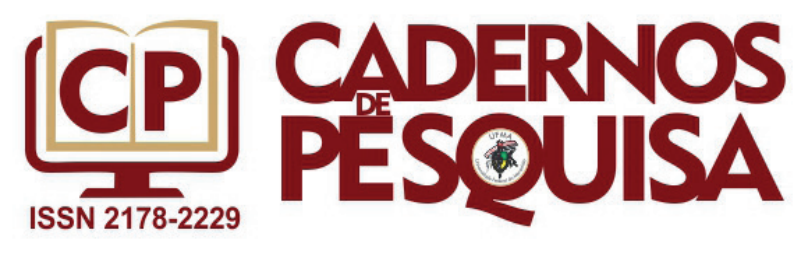

\title{
Práticas familiares de leitura e o desenvolvimento da competência leitora: uma revisão da literatura
}

\section{Family practices of reading and the development of the competence of the reader: a review of the literature}

\author{
Prácticas familiares de lectura y el desarrollo de la \\ competencia del lector: una revisión de la literatura
}

\author{
Sarah Aline Roza ${ }^{1}$ \\ http://orcid.org/0000-0002-7777-7155
}

Girlane Moura Hickmann ${ }^{2}$

http://orcid.org/0000-0002-5960-7478

Sandra Regina Kirchner Guimarães ${ }^{3}$

http://orcid.org/0000-0003-3441-0983

\begin{abstract}
Resumo: Este estudo revisou os resultados de pesquisas empíricas que focalizaram as relações entre as práticas familiares de leitura e o desenvolvimento da competência leitora das crianças. A revisão sistemática seguiu as etapas postuladas pelo protocolo PRISMA. Após a aplicação dos critérios de inclusão e exclusão estabelecidos, foram selecionadas e analisadas 38 pesquisas, a maioria (34 pesquisas) encontradas nas seguintes bases de dados: ERIC, Periódicos CAPES e PsycINFO. Além dessas, foram incluídos na revisão outros quatro estudos citados pelas pesquisas selecionadas. Eles demonstraram estar de acordo com os critérios estabelecidos. Os resultados das pesquisas mostram relações entre aspectos sociodemográficos - tais como o nível socioeconômico e a instrução acadêmica dos pais - com a aprendizagem inicial e aperfeiçoamento da leitura. Entre as conclusões dos estudos, identificou-se que o estímulo à leitura proveniente do ambiente familiar está relacionado com o letramento e a competência em leitura. Conhecer essas relações pode subsidiar políticas públicas voltadas à educação infantil e aos anos inicias do Ensino Fundamental, bem
\end{abstract}

1 Universidade Federal do Paraná (UFPR). Curitiba - PR, Brasil. ORCID: https://orcid.org/0000-0002-77777155. E-mail: sarah.a.roza@gmail.com.

2 Universidade Federal do Paraná (UFPR). Curitiba - PR, Brasil. ORCID: http://orcid.org/0000-0002-59607478. E-mail: girlanehickmann@gmail.com.

3 Universidade Federal do Paraná (UFPR). Curitiba - PR, Brasil. ORCID: http://orcid.org/0000-0003-34410983. E-mail: srkguimaraes@uol.com.br. 
como o desenvolvimento de atividades curriculares que devem ser destacadas nos projetos político-pedagógicos das escolas.

Palavras-chave: Leitura. Práticas familiares de leitura. Competências de leitura em crianças.

\begin{abstract}
This study aimed to review the findings of researches that focused on the relationship among family reading practices and the development of reading competence in children. The systematic review developed in this study followed the steps suggested by the PRISMA protocol. Thus, after applying the established inclusion and exclusion criteria, 38 studies were selected and analyzed, most of them (34 papers) were found in the following databases: ERIC, Periódicos CAPES and PsycINFO. In addition, four other studies were included in the review, because they were cited by the selected studies and were related to the established criteria. The results suggested a relationship between sociodemographic aspects - such as the socioeconomic level and the academic instruction of the parents - with the initial learning and improvement of the reading. Moreover, the studies also state that when parents encourage their children to reading it may be associated with a higher level of literacy and other reading competence. Therefore, these results may support public policies designed to early childhood education and to the early years of elementary education, as well as the development of curricular activities that should be highlighted in the political-pedagogical projects of schools.
\end{abstract}

Keywords: Reading. Family practices of reading. Reading skills in children.

Resumen: Este estudio revisó los resultados de investigaciones empíricas que enfocaron las relaciones entre las prácticas familiares de lectura y el desarrollo de la competencia lectora de los niños. La revisión sistemática siguió las etapas postuladas por el protocolo PRISMA. Después de la aplicación de los criterios de inclusión y exclusión establecidos, se seleccionaron y analizaron 38 investigaciones, la mayoría (34 artículos) encontrados en las siguientes bases de datos: ERIC, Periódicos CAPES y PsycINFO. Además de estas, se incluyeron en la revisión otros cuatro estudios, que fueron citados por las encuestas seleccionadas y demostraron estar de acuerdo con los criterios establecidos. Los resultados de las encuestas muestran relaciones entre aspectos sociodemográficos-tales como el nivel socioeconómico y la instrucción académica de los padres- con el aprendizaje inicial y el perfeccionamiento de la lectura. Entre las conclusiones de los estudios se identificó que el estímulo a la lectura proveniente del ambiente familiar está relacionado con el letramento y la competencia en lectura. Conocer estas relaciones puede subsidiar políticas públicas dirigidas a la educación infantil ya los años iniciales de la enseñanza fundamental, así como el desarrollo de actividades curriculares que deben ser destacadas en los proyectos político-pedagógicos de las escuelas.

Palabras clave: Lectura. Prácticas familiares de lectura. Competencias de lectura en niños.

\title{
1 INTRODUÇÃO
}

O contexto de desenvolvimento familiar e as práticas familiares de leitura e seus impactos na leitura de crianças e adolescentes, como assunto de pesquisa, é relativamente recente (PURCELL-GATES, 2000). Embora as práticas familiares de leitura possam existir há séculos, apenas nas últimas décadas a temática ganhou destaque para pesquisadores, políticos, teóricos, pais e professores (ARAM; LEVIN, 2011). No cenário brasileiro, ainda há lacunas tanto em relação à socialização das investigações, quanto à compreensão do alcance desses estudos e das temáticas associadas a eles, permanecendo pouco conhecidos do público diretamente afetado, notadamente pais e professores.

Nas últimas décadas, estudos têm investigado como e quanto a participação em atividades de leitura em contextos extraescolares pode impactar a aprendizagem inicial 
e o aperfeiçoamento da leitura, bem como a compreensão de textos no contexto escolar. (BURGESS; HECHT; LANIGAN 2002; KAMIL et. al., 2000; VAN STEENSEL, 2006). Destaca-se que a compreensão de textos é uma competência complexa e multifacetada, a qual se relaciona ao processo de leitura. Além disso, a habilidade de compreender textos é também adquirida e desenvolvida por intermédio das interações sociais e culturais com o ambiente, não sendo, portanto, uma capacidade genética (ROAZZI, HODGES; NOBRE, 2019). Desse modo, as interações que ocorrem no contexto familiar podem figurar como importante contribuição para as competências de leitura e de compreensão de textos orais e escritos.

A importância da leitura e da compreensão de textos - tanto para o sucesso educacional nas diferentes disciplinas escolares como para toda e qualquer relação social e exercício da cidadania - indica a necessidade de se entender mais sobre as possíveis influências que as práticas de leitura, especialmente as realizadas em contexto familiar, exercem sobre o processo de ensino-aprendizagem da leitura que tem o seu espaço de ocorrência na escola.

À luz de evidências de que as crianças provenientes de níveis socioeconômicos mais baixos são mais propensas a exibir atrasos na linguagem e no aprendizado ao entrar na escola (HOFF, 2003; RODRIGUEZ et al., 2009), são necessárias pesquisas adicionais para entender por que essas diferenças existem e como apoiar os pais na provisão de um ambiente que promova habilidades prévias ao aprendizado da leitura e da compreensão de textos. Por outro lado, há pesquisadores que sugerem que todas as crianças, independentemente do status socioeconômico (BENNETT; WEIGEL; MARTIN, 2002; RAIKES et al., 2006), se beneficiam de ambientes letrados ${ }^{4}$ e de incentivo para a compreensão de textos.

De acordo com as metas da Agenda Sustentável da UNESCO (2017) para 2030, uma abordagem que integre a família e a escola - com vistas ao aprendizado tanto da leitura e da escrita como outras competências - figura como um dos maiores desafios a serem superados, especialmente em países emergentes como o Brasil. A meta é quebrar as barreiras que existem entre o lar, a escola e a comunidade, buscando a criação e o incentivo de ambientes que estimulem e impulsionem práticas de leitura, compreensão e produção de textos, independentemente dos níveis socioeconômicos. Para tanto, será necessário promover uma cultura de cooperação entre família e escola, na qual as dificuldades de uma ou de outra não sejam ignoradas, mas trabalhadas.

Spinillo (2013) destaca que os conhecimentos prévios (conhecimentos que os indivíduos possuem) utilizados pelos leitores para a leitura e compreensão de textos não são

\footnotetext{
4 Autores como Viana e Cadime (2017) discorrem sobre os ambientes que facilitam a aprendizagem da leitura e da escrita, salientando que o letramento emerge na vida da criança antes mesmo de ela iniciar a escolarização formal e utilizam o termo "letramento emergente" para se referirem ao desenvolvimento e às aprendizagens de crianças pequenas. Nesse contexto, as autoras destacam que as noções das crianças sobre leitura dependem, consideravelmente, do meio em que vivem.
} 
os mesmos, e que o contexto sociocultural é importante para a construção dos sentidos atribuídos ao texto. Nessa perspectiva, é possível dizer que as crianças entram na escola com diferentes níveis de conhecimento de mundo. Essas diferenças podem afetar a aprendizagem da leitura e o crescente domínio dos níveis de compreensão leitora (por exemplo: compreensão de aspectos explícitos e compreensão de aspectos implícitos no texto). Nesse sentido, é que o contexto familiar, o acesso que as crianças têm a materiais de leitura e os hábitos de leitura dos pais se constituem como fatores que podem influenciar o desenvolvimento da leitura e da escrita dos estudantes e se tornam importantes elementos a serem investigados.

Ademais, considera-se relevante salientar que a investigação científica na área da leitura tem focalizado sua atenção na aprendizagem inicial e aperfeiçoamento da leitura e da escrita (BENNETT; WEIGEL; MARTIN, 2002; GUIMARÃES; BRANCO, 2010), bem como na identificação de dificuldades nas habilidades subjacentes à aprendizagem da leitura (COMPTON, 2002; GUIMARÃES, 2003). Entretanto, poucas pesquisas investigam o ambiente alfabetizador em casa e o desenvolvimento de habilidades fundamentais para a aprendizagem da leitura (BURGESS; HECHT; LONIGAN, 2002).

Assim, é preciso desvelar as práticas que estão sendo engendradas nos contextos familiares dos estudantes, bem como as repercussões escolares dessas práticas, para então propor alternativas pedagógicas que possam fornecer aos alunos maneiras de superar as possíveis lacunas na estimulação para a leitura. Dessa forma, acredita-se que a quantidade e a qualidade de adequadas atividades voltadas para o desenvolvimento da leitura - atividades que contemplem suas dimensões social, linguística e cognitiva - podem evitar, ou ao menos, atenuar as dificuldades na aprendizagem inicial da leitura e na compreensão de textos. Salienta-se que diferentes habilidades linguístico-cognitivas podem ser requisitadas no desenvolvimento da leitura, em função do momento da trajetória escolar das crianças (CORREA; MOUSINHO, 2013). Cada fase requer uma nova organização dessas habilidades, bem como um novo desenho quanto às suas influências na performance em leitura.

Nessa perspectiva, a UNESCO (2017) destaca a necessidade de pesquisas que possam contribuir com o entendimento dos processos de ensino-aprendizagem da leitura e da compreensão e produção de textos por parte de crianças em desenvolvimento, investigando as contribuições dos ambientes extraescolares, notadamente do ambiente familiar para as aprendizagens escolares. Além disso, estas pesquisas devem fornecer subsídios para que tanto os pais como os professores compreendam a importância deste conjunto de contribuições (saberes advindos de diferentes contextos) para o desenvolvimento acadêmico.

É nesse contexto que esta revisão se insere, propondo-se a examinar os estudos que tiveram como objetivo investigar a associação entre as práticas familiares de leitura e o desenvolvimento da leitura (aprendizagem inicial da leitura e da compreensão de textos) 
entre os estudantes do Ensino Fundamental (Educação Básica) oriundos das famílias investigadas. Assim, pretende-se com esta revisão buscar elementos de resposta para duas questões principais: 1) Qual é o nível de significância da relação entre as práticas familiares de leitura e a aprendizagem de habilidades de leitura pelos estudantes oriundos dessas famílias (competências de letramento emergente, alfabetização, aprendizagem inicial da leitura e compreensão de texto)? 2) Como o contexto sociocultural e econômico das famílias impacta a aprendizagem inicial e no aperfeiçoamento da leitura entre os estudantes do Ensino Fundamental (Educação Básica)?

\section{MÉTODO}

A presente revisão sistemática de literatura foi elaborada a partir das recomendações estabelecidas pelo PRISMA (Principais Itens para Relatar Revisões Sistemáticas e Meta-análises), conforme Galvão e Pansani (2015). Dessa forma, efetuou-se um levantamento de artigos no Portal de Periódicos CAPES, no Centro de Informações de Recursos e fontes em Educação (Education Resources Information Center - ERIC) e na Base de dados no campo da Psicologia, desenvolvido pela Sociedade Americana de Psicologia - (PsycINFO). A pesquisa aconteceu no período de agosto de 2017 a abril de 2018, utilizando os descritores: "práticas familiares de leitura", "leitura ou compreensão de textos" e "nível sócio econômico", nos idiomas português, inglês e espanhol.

Os critérios para inclusão foram: pesquisas empíricas envolvendo os descritores selecionados; estudos envolvendo crianças e seus familiares (focalizando nos pais); pesquisas de distintos países, desde que publicadas em português, inglês ou espanhol. Foram excluídos estudos que não abordavam as práticas familiares em associação com a leitura e/ou compreensão leitora em crianças; que envolviam outras habilidades acadêmicas que não as relacionadas à leitura; que apresentavam questões familiares relacionas a algum tipo de distúrbio ou problema na leitura; que associaram o status econômico a questões escolares gerais que não a leitura e compreensão de textos; que utilizaram como metodologia o estudo de caso ou pesquisa bibliográfica.

Destaca-se que, além de selecionar as pesquisas e artigos a partir dos critérios de inclusão e exclusão descritos, foi verificada a repetição dos trabalhos em diferentes bases de dados e, nesses casos, os estudos não foram computados duas vezes. Assim, foram selecionados 38 artigos, sendo 20 do ERIC, oito dos Periódicos CAPES, seis do PsycINFO. Quatro outros artigos foram adicionados manualmente, a partir da leitura das referências encontradas nos artigos selecionados, os quais não constavam nas bases de dados escoIhidas nesta pesquisa (Quadro 01). 
Quadro 01 - Total dos artigos encontrados e artigos selecionados nesta revisão de literatura

\begin{tabular}{l|ccccc}
\hline \multirow{2}{*}{\multicolumn{1}{c|}{ Descritores }} & \multicolumn{4}{c}{ Fonte de pesquisa } \\
\cline { 2 - 6 } & ERIC & $\begin{array}{c}\text { Periódicos } \\
\text { Capes }\end{array}$ & PsycINFO & Manual & Total \\
\hline Práticas familiares de leitura & 63 & 12 & 31 & 3 & 109 \\
Leitura e Compreensão de textos & 73 & 35 & 71 & 1 & 180 \\
Nível sócio econômico & 39 & 27 & 7 & - & 73 \\
\hline Total & 175 & 74 & 109 & 4 & 362 \\
\hline Selecionados após verificação das & 20 & 8 & 6 & 4 & 38 \\
relações entre as variáveis & & & & & 3 \\
\hline
\end{tabular}

Fonte: Elaborado pelas autoras (2018).

Além dos artigos selecionados, foram encontradas revisões de literatura relacionadas ao tema investigado neste artigo. Uma revisão sistemática da literatura sobre o impacto do ambiente familiar no desempenho escolar (MAHENDRA; MARIN, 2015), a qual não aborda a leitura de modo específico; e uma revisão sistemática da literatura sobre home literacy e alfabetização (MOTA, 2014), a qual se preocupa em conceituar e contextualizar o tema home literacy, fazendo uma associação dessa definição com a alfabetização, focalizando principalmente na habilidade de escrita desenvolvida por crianças. Portanto, as revisões encontradas não tiveram o mesmo propósito deste trabalho, ou seja, revisar e analisar os dados de pesquisas acerca dos estudos que focalizaram as práticas familiares de leitura e seu impacto na leitura e compreensão textual em crianças.

\section{RESULTADOS E DISCUSSÃO}

Esta revisão de literatura focalizou o tema "práticas familiares de leitura e o desenvolvimento da leitura (aprendizagem inicial da leitura e compreensão de textos) em crianças". Ademais, buscaram-se dados de pesquisa mostrando como o contexto sociocultural - com ênfase nos aspectos socioeconômicos - impacta a performance em leitura e compreensão de textos. Em função disso, inicialmente apresenta-se uma caracterização geral dos estudos, destacando os períodos de publicação, as temáticas abordadas e o público-alvo dos estudos elencados. Além disso, são elencadas as abordagens mais utilizadas nas pesquisas sobre práticas familiares de leitura e o impacto dessas práticas sobre o desenvolvimento da leitura entre as crianças. Em seguida, são apresentadas seis subseções nas quais são detalhados os estudos envolvendo cada uma das temáticas relacionadas às práticas familiares de leitura.

Destaca-se que, neste artigo, as práticas familiares de leitura são circunscritas ao sentido de práticas, ações e suportes familiares que podem influenciar o desenvolvimento 
da leitura e da compreensão de textos em crianças. Portanto, pretende-se verificar o que as pesquisas que abordam esse objeto de investigação têm apresentado como elementos do ambiente familiar que estimulam e impactam a leitura, incluindo o nível socioeconômico como fator presente no contexto de desenvolvimento das crianças.

O recorte dessa revisão inclui os trabalhos encontrados até abril de 2018. A primeira publicação sobre o tema data de 1994, e nos primeiros 10 anos posteriores a quantidade de artigos sobre a temática foi discreta, com 10 publicações encontradas sobre as práticas de leitura familiares e seu impacto na leitura e compreensão de textos em crianças. De 2005 a 2018, foram publicados 28 artigos envolvendo a temática, o que demonstra um interesse maior de pesquisadores em compreender de que forma as práticas desenvolvidas por pais e familiares podem influenciar o desenvolvimento da leitura e da compreensão de textos em crianças.

Assim como as demais pesquisas e estudos envolvendo o aprendizado que, potencialmente, ocorre no ambiente familiar, esta revisão confirma que os pais (mãe e pai) são investigados como a principal fonte de suporte para a aprendizagem e desenvolvimento de crianças e adolescentes no que se refere à leitura.

Primeiramente destacam-se os estudos que mostram a associação das práticas familiares de leitura com práticas de alfabetização (BENNETT; WEIGEL; MARTIN, 2002; NIKLAS; SCHNEIDER, 2015; QUADROS; SARROUB, 2016; SANTOS; ALVES, 1994; SANTOS; JOLY, 1996; SÉNÉCHAL et al., 1998; SÉNÉCHAL; LEFEVRE, 2002; SWAIN; CARA; MALLOWS, 2017).

Pesquisas relacionando a alfabetização/aprendizagem da leitura com o contexto sociocultural, conferindo ênfase nos aspectos socioeconômicos e nível de instrução dos pais também são frequentes (BALTAR; MOTA, 2016; BRITO; BROOKS-GUNN, 2001; CARDOSO; MOTA, 2015; COSTA et al. 2016; ERGÜL et al., 2017; HOFF, 2003; KIRBY; HOGAN, 2008; KORAT; KLEIN; SEGAL-DRORI, 2007; MENDIVE et al., 2017; PURCELL-GATES, 1996; RAIKES et al., 2006; SONNENSCHEIN; MUNSTERMAN, 2002; VAN STEENSEL, 2006; VASILYEVA; WATERFALL, 2011).

Estudos focalizando esforços em compreender o letramento emergente, suas definições e conceitos aplicados à prática familiar e à pesquisa também foram verificados (CRUZ et al., 2012; EDWARDS, 2014; KIM, 2009; SALVADOR; MARTINS, 2017; MATA, 2012; STORCH; WHITEHURST, 2001; VIANA; RIBEIRO; BARRERA, 2017). Além disso, outros estudos destacaram influências do contexto de desenvolvimento de acordo com o gênero (BAKER; MILLIGAN, 2016; DINIZ et al., 2014; RAIKES et al., 2006; SWALANDER; TAUBE, 2007) e de acordo com o país (BAKER; MILLIGAN, 2013; CHIU; MCBRIDE-CHANG, 2010; EVANS et al., 2010; RAIKES et al., 2006). Por último, identificou-se que alguns estudos voltados para a investigação das práticas familiares de leitura incluíram os aspectos cognitivos e a hereditariedade como variáveis a serem pesquisadas (BERGEN et al., 2016; COLON et al., 2006; ENRICONE; SALLES, 2011). 
A maioria das pesquisas envolveu crianças com idade correspondente à educação infantil. Além disso, 11 estudos investigaram crianças dos anos iniciais do Ensino Fundamental (CARDOSO; MOTA, 2015; DINIZ et al., 2014; ENRICONE; SALLES, 2011; KIRBY; HOGAN, 2008; KORAT; KLEIN; SEGAL-DRORI, 2007; PURCELL-GATES, 1996; SALVADOR; MARTINS, 2017; SANTOS; ALVES, 1994; SÉNÉCHAL et al., 1998; SÉNÉCHAL; LEFEVRE, 2002; VAN STEENSEL, 2006); oito estudos focalizaram estudantes dos anos finais, isto é, adolescentes (BALTER; MOTA, 2016; CHIU; MCBRIDE-CHANG, 2010; COLON et al., 2006; COSTA et al., 2016; DINIZ et al., 2014; SANTOS; ALVES, 1994; SWALANDER; TAUBE, 2007; VAN STEENSEL, 2006).

Diferentes delineamentos de pesquisa foram adotados pelos estudos analisados. Contudo, destacam-se os cinco estudos longitudinais. O estudo de maior duração foi iniciado em 1986 e se estendeu até o ano 2000, investigando como os aspectos socioeconômicos e de instrução dos pais influenciam a habilidade de leitura de crianças (BRITO; BROOKS-GUNN, 2001). Publicado um ano depois, outro estudo longitudinal investigou o envolvimento dos pais no desenvolvimento das habilidades de leitura nas crianças, durante um período de cinco anos (SÉNÉCHAL; LEFEVRE, 2002). Ainda em 2002, foi publicado outro estudo que analisou o impacto exercido pelo ambiente familiar sobre o desenvolvimento de habilidades relacionadas à leitura ao longo de um ano (BURGESS; HECHT; LONIGAN, 2002). Em 2009, o estudo de Rodrigues et al. investigou o papel formativo de práticas familiares de leitura durante os três primeiros anos de vida de 1046 crianças de nível socioeconômico baixo. Finalmente, o último estudo elencado (JUNG, 2016), foi realizado em larga escala - aproximadamente 21.000 crianças de escolas públicas, católicas e particulares - e coletou dados em 1998 e 1999, a partir dos quais analisou a influência das atividades familiares e atitudes das crianças em relação à escola sobre o desenvolvimento de habilidades de leitura na educação infantil.

A partir desse panorama, serão apresentados em seis subseções. Os estudos selecionados nessa revisão foram agrupados de forma a destacar como as práticas familiares de leitura se associam com outras temáticas/variáveis.

\subsection{RELAÇÃO DAS PRÁTICAS FAMILIARES DE LEITURA COM A ALFABETIZAÇÃO}

De acordo com Bennett, Weigel e Martin (2002), o modelo de família como educadora está significativamente relacionado à linguagem oral e às habilidades precursoras da alfabetização, em crianças pré-escolares com potenciais impactos sobre o desenvolvimento da leitura e escrita, nos anos iniciais do Ensino Fundamental. Como pode ser verificado na sequência, os trabalhos que focalizaram a relação das práticas familiares de leitura com a alfabetização utilizaram diferentes métodos de investigação, ou seja, identificaram-se estudos correlacionais e estudos de intervenção. 
Em um estudo de intervenção, Santos e Joly (1996) realizaram uma série de ações com os pais de crianças da educação infantil de escolas públicas e privadas. Após a intervenção, verificaram que as ações desenvolvidas impactaram, de forma significativa, o desempenho e atitude frente à leitura para ambas as realidades estudadas. Em estudo semelhante, Niklas e Schneider (2015) desenvolveram uma intervenção com as famílias de 117 crianças, no intuito de aumentar as práticas familiares de leitura. As competências dessas crianças foram verificadas antes e depois da intervenção em comparação com um grupo que não participou da intervenção. As famílias que participaram das intervenções não só melhoraram suas práticas familiares de leitura, mas também as crianças nessas famílias apresentaram maior desenvolvimento de competência linguística quando comparadas com o grupo não participante.

Publicados em inglês, dois estudos desenvolvidos por Sénéchal e colaboradores são amplamente citados e referenciados na literatura sobre práticas familiares de leitura e alfabetização, tendo em vista que mostram uma associação significativa entre o que ocorre no contexto familiar e as habilidades escolares de leitura em crianças (SÉNÉCHAL et al., 1998; SÉNÉCHAL; LEFREVE, 2002). No primeiro estudo, Sénéchal et al. (1998) verificaram, por meio de uma pesquisa com 110 crianças - pré-escolares e do $1^{\circ}$ ano do Ensino Fundamental e seus pais - que a exposição a livros de história aumenta as habilidades de linguagem. Os autores indicaram que os pais podem contribuir com a ampliação de experiências das crianças com os livros, o que reflete no desenvolvimento de habilidades essenciais para a alfabetização. Além disso, os pesquisadores verificaram associações entre as experiências de letramento/alfabetização vivenciadas em casa, ou seja, o apoio dado pelos familiares às atividades de leitura, e o posterior desenvolvimento das habilidades de leitura no contexto escolar.

O segundo estudo referido, produto de cinco anos de pesquisa, publicado por Sénéchal e LeFevre (2002), confirmou que a exposição de crianças a livros como parte do suporte familiar de leitura está relacionada ao desenvolvimento do vocabulário e de habilidades de compreensão de textos. Os autores fazem destaque para o fato de que as competências desenvolvidas pelas crianças participantes do estudo mostraram-se diretamente associadas à leitura no $3^{\circ}$ ano do Ensino Fundamental. Outro estudo longitudinal também constatou que as atividades e práticas de leitura familiares têm influência inicial e permanente em relação ao desenvolvimento da leitura e da escrita em crianças (BURGESS; HECHT; LONIGAN, 2002).

Trabalhando exclusivamente com as mães, Santos e Alves (1994) investigaram os comportamentos dos filhos referentes à leitura, desde a entrada das crianças no $1^{\circ}$ ano até $08^{\circ}$ ano do Ensino Fundamental. Verificaram que gibis e livros de histórias são os materiais mais utilizados pelos professores, tanto na alfabetização como no processo de aperfeiçoamento da leitura e da alfabetização. Segundo seus relatos, essas leituras motivam as crianças. Nesta perspectiva, as autoras destacam o importante papel dos familiares 
que podem fornecer adicionais indicações de leitura, tanto para as crianças quanto para os adolescentes.

Outro estudo que forneceu evidências sobre a importância das práticas familiares de leitura para o desenvolvimento da capacidade leitora dos estudantes foi realizado por Swain, Cara e Mallows (2017). Os autores exploraram as práticas de leitura de 254 famílias de crianças pré-escolares. Uma das principais conclusões do estudo foi que os alunos com maior facilidade para alfabetização e subsequente melhor desempenho em leitura têm mais experiências de leituras a partir da família, ou seja, ouvem seus pais lerem, frequentam a biblioteca com eles, observam quando estão lendo para si mesmos e outros.

Por último, é importante mencionar que a temática foi investigada também com participantes de um grupo étnico de refugiados, conhecidos como Karen, comunidade nativa do sudeste da Ásia, em Mianmar. Quadros e Sarroub (2016), autores do estudo, investigaram as relações entre as experiências das crianças e suas mães com a leitura e a alfabetização, sem desconsiderar aspectos socioculturais. Os resultados obtidos são produto da observação participante dos pesquisadores e destacam o envolvimento das mães com a memorização e com as práticas de alfabetização por meio da leitura em voz alta (recitação) de seus filhos.

\subsection{RELAÇÕES ENTRE O NÍVEL SOCIOCULTURAL DAS FAMÍLIAS E O DESENVOL- VIMENTO DE COMPETÊNCIAS ASSOCIADAS À LEITURA}

Muitos estudos têm demonstrado que aspectos individuais das crianças, como o nível socioeconômico de sua família de origem e nível de instrução dos pais, podem impactar o desenvolvimento de competências associadas à leitura (NEUMAN; DICKINSON, 2011). Nesse contexto, torna-se uma tarefa importante verificar a forma pela qual as pesquisas investigam as práticas familiares de leitura em distintos contextos socioeconômicos. A análise dessas pesquisas pode auxiliar na compreensão de possíveis variabilidades presentes no desenvolvimento da capacidade de leitura das crianças.

Tomando como fio condutor de apresentação dos trabalhos revisados a cronologia das publicações, serão citados primeiramente aqueles realizados com participantes estrangeiros (que não falam português). Em seguida, serão apresentados os três estudos realizados com falantes do português. Assim, o primeiro estudo selecionado foi realizado por Purcell-Gates (1996), que examinou práticas de leitura em 20 residências de diferentes níveis socioeconômicos. O princípio alfabético e formas específicas de leitura e escrita apareceram mais frequentemente em lares onde os pais liam e escreviam com proficiência, ou seja, possuíam elevados níveis de instrução e dominavam formas complexas de discurso. A partir desses achados, os autores sugerem que o nível de instrução dos pais tem relação tanto com a aprendizagem como com o desenvolvimento da leitura das crianças. 
Selecionaram-se também cinco estudos publicados entre 2001 e 2008. O primeiro, publicado por Brito e Brooks-Gunn (2001), mostrou que dentre os elementos do ambiente familiar que podem impactar o letramento, a alfabetização e a leitura, destaca-se a linguagem expressiva materna. Para chegar a essa conclusão, os autores acompanharam 126 famílias. Os dados desse acompanhamento mostraram que as variáveis socioeconômicas e o nível de instrução dos pais influenciam fortemente a atividade de leitura e o desenvolvimento de competências relacionadas à leitura em crianças. Além disso, os autores verificaram que a qualidade das oportunidades de aprendizagem e apoio emocional em casa são mais fortes para os filhos de mães com mais escolaridade do que para os filhos de mães que não completaram a educação básica.

No ano seguinte, no intuito de compreender melhor as práticas familiares e suas implicações na leitura de crianças, Sonnenschein e Munsterman (2002) investigaram 30 famílias de pré-escolares, caracterizadas por uma das seguintes quatro características: baixo nível socioeconômico, ter ascendência africana, ser europeu ou ser mestiço. Foram codificadas práticas de leitura (tipos de comentários feitos durante a leitura e qualidade afetiva da interação durante a leitura). A qualidade da interação afetiva foi o preditor mais poderoso para as motivações de leitura das crianças. Não foram encontradas outras associações significativas. Nesse estudo, as questões socioeconômicas e étnica não apontaram diferenças significativas para o desempenho e desenvolvimento da leitura em crianças. Entretanto, Hoff (2003) testou a hipótese de que crianças que diferem em relação ao nível socioeconômico vivenciam diferentes práticas familiares de leitura e, consequentemente, têm diferentes escores e relações com a leitura. A comparação entre dois grupos com 30 mães e filhos de diferentes níveis socioeconômicos demonstrou que as crianças pertencentes a estratos socioeconômicos mais elevados apresentaram um vocabulário mais produtivo, maior facilidade para o aprendizado da leitura, bem como melhores resultados na leitura.

Além disso, na investigação realizada com um expressivo número de mães, ou seja, 2.581 mães de diferentes níveis socioeconômicos, Raikes et al. (2006) verificaram que mães brancas e com níveis socioeconômicos mais elevados leem mais do que mães hispânicas ou afro-americanas de níveis socioeconômicos mais baixos. Esses resultados mostram que as desigualdades sociais, relacionadas ao nível socioeconômico e de instrução das mães dos alunos, acarretam prejuízos nas oportunidades de aprendizagem às crianças que estão em fase escolar, especialmente em relação à leitura. Neste mesmo ano, Van Steensel (2006) publicou estudo sobre as relações entre o ambiente familiar e o desenvolvimento da alfabetização e leitura em crianças de séries iniciais do Ensino Fundamental, focalizando em como essa relação ocorre com base no nível socioeconômico. A partir de levantamento demográfico e testes de leitura, o autor constatou que crianças de níveis socioeconômicos mais elevados eram, em geral, mais estimuladas. Em decorrência disso, obtinham melhores escores em testes de leitura. Entretanto, as crianças oriundas de grupos de baixo nível 
socioeconômico demonstraram grande variabilidade nos resultados nos testes de leitura, ou seja, desde baixos até altos níveis de pontuação.

Korat, Klein e Segal-Drori (2007) investigaram 94 crianças, sendo 47 de nível socioeconômico baixo e 47 de nível socioeconômico alto, com o propósito de verificar se a mediação de leitura materna e o ambiente familiar de alfabetização variam em função dos estratos socioeconômicos. As interações entre mães e filhos foram filmadas e suas expressões verbais foram decodificadas. As crianças de famílias com níveis socioeconômicos mais elevados demonstraram uma alfabetização mais eficiente e melhores níveis de leitura em comparação com crianças provenientes de níveis socioeconômicos baixos. Já o estudo publicado por Kirby e Hogan (2008) mostra as diferenças entre as práticas familiares de leitura de crianças provenientes de diferentes ambientes socioeconômicos e os escores obtidos por essas crianças quando submetidas a uma bateria de medidas de leitura. Os resultados evidenciaram que, dentre as principais influências na leitura de crianças, o nível socioeconômico, o número de livros em casa e a escolaridade dos pais foram os mais decisivos para um bom engajamento com a leitura $\mathrm{e}$ níveis mais altos de habilidades leitoras. Um estudo longitudinal realizado em escolas públicas e privadas verificou que a presença dos pais em práticas de leitura é um importante preditor para níveis mais elevados de leitura e escrita nas séries inicias (JUNG, 2016).

Publicados na década seguinte, foram encontrados dois estudos em inglês, um realizado com participantes de nacionalidade turca e o outro com chilenos. Assim, Ergül et al. (2017) publicaram estudo em que investigaram as diferenças entre ambientes familiares de alfabetização de crianças provenientes de três diferentes níveis socioeconômicos na Turkia. Para tanto, os pesquisadores utilizaram um Questionário de Alfabetização Familiar e realizaram um levantamento sociodemográfico. Os resultados indicaram diferenças significativas entre os três grupos, sendo que as crianças com níveis socioeconômicos mais elevados obtiveram escores mais altos em todas as questões do questionário. Esses resultados destacam desvantagens para os grupos de níveis socioeconômicos mais baixos, sugerindo que os educadores devem pensar formas de dar suporte a essas famílias em situação de desvantagem econômica. O outro estudo foi publicado por Mendive et al. (2017). Foram investigadas as práticas de leitura e alfabetização com 989 mães chilenas de famílias que tinham filhos na educação infantil, observando como essa interação ocorre diante do nível socioeconômico e da instrução das mães dessas crianças. Os resultados sugerem a necessidade de intervenções junto a mães de famílias com baixo nível socioeconômico ou que não possuam o Ensino Médio completo, como forma de aprimorar as práticas educativas que as crianças vivenciam em casa, antes de começarem a frequentar a educação pré-escolar.

Na década atual, as relações entre o nível sociocultural das famílias e o desenvolvimento da leitura também têm sido investigadas por pesquisadores brasileiros (BALTAR; MOTA, 2016; CARDOSO; MOTA, 2015; COSTA et al. 2016). Cardoso e Mota (2015) publicaram o resultado do estudo no qual investigaram as práticas familiares com crianças de níveis socioeconômicos elevado e baixo, relacionando-as com o aprendizado da leitura. 
Foram aplicados questionários e testes de consciência fonológica e vocabulário. Os resultados revelaram que o estímulo em casa é diferente para estudantes de escolas públicas e privadas e que as crianças das escolas privadas tiveram escores significativamente mais elevados do que os estudantes de escola pública nos testes de leitura.

No ano seguinte, Baltar e Mota (2016) publicaram um estudo realizado no Rio de Janeiro, cujos participantes foram 31 adolescentes e suas famílias. De acordo com os resultados do estudo tanto a motivação quanto a performance elevada em leitura estão associadas ao material que adolescentes possuem em casa. Corroborando estes resultados, o estudo publicado por Costa et al. (2016) mostra que o suporte familiar e as atitudes dos pais frente à leitura são decisivos para um bom desempenho em leitura, não apenas em crianças, mas também em adolescentes.

\subsection{RELAÇÃO DAS PRÁTICAS FAMILIARES DE LEITURA COM O LETRAMENTO EMERGENTE}

O letramento emergente faz referência ao processo fundamental de aquisição de competências específicas da linguagem que se desenvolvem antes da entrada na educação formal (VIANA; RIBEIRO; BARRERA, 2017). As pesquisas sobre as práticas familiares de leitura, em sua maioria, focalizam sua atenção na educação infantil e nos resultados de influências que os pais exercem sobres seus filhos nessa etapa e que resultam em benefícios para as crianças durante a alfabetização e o desenvolvimento de competências de leitura no período de entrada na escolarização formal.

Durante a realização de seu estudo, Edwards (2014) verificou que mães que se envolveram em atividades de letramento com seus filhos, por ocasião de interações de leitura compartilhada, levaram essas crianças a mostrar consciência de habilidades da linguagem escrita, mesmo antes da entrada no Ensino Fundamental. Ainda sobre a relevância atribuída ao estímulo familiar para a leitura, Storch e Whitehurst (2001) destacam que as famílias têm papel central no desenvolvimento do letramento emergente em crianças pré-escolares, embora tenham verificado que crianças provenientes de famílias de níveis socioeconômicos baixos tenham menor vivência de práticas de letramento. A partir destes resultados sugerem que estas crianças precisam receber mais estímulos para a aprendizagem da leitura.

Kim (2009) investigou em 192 crianças coreanas e suas famílias o impacto das práticas familiares de leitura e verificou que a leitura familiar está positivamente associada a habilidades de letramento emergente. Na mesma linha, Mata (2012) verificou que as famílias desempenham um papel importante no desenvolvimento da literacia, ou seja, as práticas familiares de leitura têm impacto na aprendizagem e desenvolvimento das crianças, pois o ambiente familiar se soma ao ambiente escolar e propicia diferentes estímulos.

Cruz et al. (2012) descrevem os efeitos de um programa de estímulo à interação entre pais e crianças pré-escolares durante a leitura. As atividades produziram impacto significativo 
nas habilidades de leitura e de escrita nas crianças, que após a participação no programa demonstraram estar mais preparadas para a alfabetização e educação formal. Os autores destacam que a participação no programa propiciou uma evolução da atenção dos pais para o impresso e uma mudança na qualidade afetiva das interações entre pais e filhos.

Mais recentemente, Salvador e Martins (2017) desenvolveram uma pesquisa com crianças da educação infantil e do $1^{\circ}$ ano do Ensino Fundamental, em Lisboa, com o intuito de verificar práticas familiares de leitura e seu impacto no letramento. Nesse contexto, descobriram que as práticas parentais de letramento relacionadas ao entretenimento, tais como leitura por prazer, são responsáveis por predizer a aprendizagem inicial da leitura de crianças em processo de alfabetização.

\subsection{AS PRÁTICAS FAMILIARES DE LEITURA E A COMPARAÇÃO POR GÊNERO}

Ao analisar os trabalhos que investigaram as práticas familiares de leitura, identificaram-se diferenças quanto aos estímulos ofertados às crianças em função do gênero. Nesta perspectiva, Baker e Milligan (2013) compararam o investimento dos pais em leitura e desenvolvimento de outras habilidades em crianças e adolescentes. Os resultados demonstram que, quando se trata de práticas de leitura, os pais investem mais nas filhas, o que leva as meninas a obterem melhores escores na leitura e em outras habilidades relacionadas à leitura. Sobre maior investimento das práticas familiares de leitura envolver as meninas, Raikes et al. (2006) verificaram que as chances de uma menina ser alvo de leitura diária é bem maior do que os meninos, especialmente as primogênitas.

Além disso, foram identificadas pesquisas que analisaram o desempenho em testes de leitura de acordo com o gênero dos participantes. Assim, Diniz et al. (2014) investigaram como o contexto de desenvolvimento familiar e o gênero podem influenciar o desempenho escolar na leitura em crianças e adolescentes. Os 627 participantes responderam um teste de performance escolar e participaram de entrevistas também para investigação de seus conhecimentos. Os resultados apontaram que o contexto de desenvolvimento impacta a leitura e escrita do $1^{\circ}$ ao $8^{\circ}$ ano do Ensino Fundamental. Além disso, as meninas alcançaram aumentos mais significativos na escrita em comparação com os meninos. Ainda sobre o desempenho de meninas, o estudo de Swalander e Taube (2007) revelou que as meninas têm uma atitude de leitura mais positiva, leem melhor diferentes gêneros de texto e apresentam um autoconceito verbal mais positivo em comparação com os meninos.

\subsection{AS PRÁTICAS FAMILIARES DE LEITURA E A COMPARAÇÃO ENTRE PAÍSES}

Alguns dos artigos selecionados tiveram como objetivo comparar, em diferentes países, as práticas familiares de leitura e seus impactos na competência de leitura das crianças. Chiu e McBride-Chang (2010) pesquisaram como as famílias e suas características 
impactam a alfabetização e a leitura das crianças de 41 macrossistemas (contextos econômicos e culturais), isto é, de 41 países diferentes. A partir da aplicação de questionários e testes de leitura, os resultados apontaram que os melhores desempenhos em leitura eram demonstrados por crianças que tinham os dois pais casados e morando com elas, nível socioeconômico médio ou elevado, muitos livros em casa, comunicação cultural em casa e poucos irmãos. No entanto, em países mais ricos, a comunicação cultural em casa mostrou-se mais fortemente associados aos escores de leitura, independentemente da presença dos dois pais. Em países com grande desigualdade, o nível socioeconômico foi o fator que obteve uma ligação mais forte com o desempenho em leitura.

Também em 2010, Evans et al. empreenderam uma pesquisa sobre a influência familiar na aprendizagem da leitura por crianças de 27 países. Os resultados destacam que o nível educacional e a cultura acadêmica dos pais, bem como o fato de a família ter vários livros em casa são fatores que aumentam muito o nível de desempenho em leitura das crianças. Esses efeitos foram encontrados não apenas em países da Europa Ocidental e suas extensões no exterior, mas também na Europa Oriental, na Ásia, na América do Sul e na África do Sul.

\subsection{PRÁTICAS FAMILIARES DE LEITURA, ASPECTOS COGNITIVOS E HEREDITA- RIEDADE}

Alguns estudos que investigaram as práticas familiares de leitura incluíram os aspectos cognitivos e a hereditariedade como variáveis a serem pesquisadas. Colon et al. (2006) analisaram habilidades de leitura em adolescentes e associações dessas com aspectos do histórico familiar. Embora os autores não tenham encontrado associações entre aspectos cognitivos e habilidades de leitura, eles destacaram que crianças com histórico de dificuldades familiares de leitura têm cinco vezes mais chances de apresentar uma leitura empobrecida e outras dificuldades nas competências leitoras.

Também buscando indícios da influência da hereditariedade sobre a capacidade de leitura, Bergen et al. (2016) realizaram um estudo que teve como participantes tríades constituídas por pai, mãe e filho. Os pesquisadores verificaram que, embora as práticas sociais familiares, isto é, as práticas associadas ao convívio, estivessem correlacionadas com a leitura das crianças, nenhum aspecto da capacidade cognitiva dos pais foi significativamente preditor da habilidade de leitura dos filhos, apenas o número de livros em casa.

No Brasil, o estudo de Enricone e Salles (2011) analisou as relações entre fatores psicossociais familiares e o desempenho em leitura e escrita em 29 famílias de estudantes do $2^{\circ}$ ano do Ensino Fundamental. A pesquisa utilizou a avaliação neuropsicológica. Os resultados apontaram para relações complexas entre fatores familiares e habilidades de leitura, havendo associações entre as dificuldades dos pais, o histórico de repetência familiar e o índice de dificuldades das crianças. Por outro lado, o histórico de sucesso familiar 
na leitura relaciona-se com o sucesso na trajetória de aprendizagem da criança. Embora as autoras destaquem que o contexto familiar é uma variável importante para a aprendizagem da leitura, não apresentam resultados que revelam aspectos de hereditariedade no desenvolvimento das habilidades de leitura.

\section{CONSIDERAÇÕES FINAIS}

Os artigos selecionados para esta revisão não relacionam as práticas familiares de leitura com as mesmas variáveis, pois investigam diferentes aspectos relacionados ao desenvolvimento da leitura. Alguns verificaram como as práticas familiares de leitura impactam aspectos da alfabetização, outros focalizaram como os aspectos socioeconômicos e de instrução dos pais podem influenciar em maior ou menor escala as competências leitoras. Destacaram-se ainda: estudos relacionando tais práticas ao letramento emergente; estudos sobre como as práticas de leitura dos pais estão relacionadas ao gênero das crianças; diferenças entre as práticas de familiares de acordo com diferentes países e, finalmente, identificaram-se estudos que avaliaram como aspectos cognitivos e hereditários permeiam a relação entre práticas familiares de leitura e desempenho leitor em crianças.

Quanto ao método dos estudos, foi possível verificar a utilização de distintos instrumentos de coleta de dados, como testes de leitura, escalas e questionários. Os resultados expressam diferentes medidas e nem sempre são comparáveis entre si. Destaca-se também o fato de que pesquisas sobre práticas familiares de leitura e seus impactos em competências de leitura geralmente têm como público-alvo de pesquisa crianças pré-escolares e de séries iniciais do Ensino Fundamental. Poucos estudos examinaram essas relações em adolescentes. O levantamento de dados socioculturais apareceu como um elemento importante: grande parte das pesquisas buscou comparar diferentes níveis de instrução e socioeconômico dos pais, na tentativa de verificar potenciais distinções nas habilidades das crianças.

O desenvolvimento da alfabetização demonstrou ter associações significativas com as práticas familiares de leitura. Desse modo, crianças com pais que estimulam a leitura em casa, durante a educação infantil ou mesmo antes da entrada na escola, seja na educação infantil ou no ensino formal, demonstram mais facilidade para desenvolver competências de leitura.

Pesquisadores e estudiosos da área da leitura têm acumulado evidências de que o nível socioeconômico está associado a diferenças no desempenho de crianças em relação à leitura, à alfabetização e ao letramento emergente. De acordo com os estudos apresentados na seção dos resultados, isso ocorre principalmente porque pais de diferentes níveis socioeconômicos tendem a prover para seus filhos distintas experiências linguísticas. Ademais, pais de estratos socioeconômicos mais elevados tendem a ter uma instrução mais elevada. Além de terem mais livros em casa, fazem leitura para as crianças e estabelecem 
variadas interações durante essas práticas de leitura, entre elas interações linguísticas e emocionais.

Nesse contexto, Mendive et al. (2017) sugerem a importância de intervenções junto às famílias com baixo nível socioeconômico ou de pais que não possuam o Ensino Médio completo. De acordo com os pesquisadores, essa seria uma alternativa para incentivar e melhorar práticas educativas envolvendo a leitura. À medida que as crianças são estimuladas em casa, elas podem desenvolver habilidades de leitura antes de começarem a frequentar a educação formal, seja na pré-escola ou nas séries iniciais do Ensino Fundamental.

O tema do letramento emergente e o da alfabetização contribuem para associações importantes entre práticas familiares de leitura e desenvolvimento das habilidades leitoras em crianças. O letramento emergente está associado à alfabetização, porém alguns estudos enfatizam o primeiro e outros a segunda, embora sempre estabeleçam a continuidade e ligação entre esses dois processos, especialmente para as crianças da Educação Infantil, principal público alvo dessas pesquisas.

Pesquisas de intervenção que tiveram pais como participantes mostraram o impacto da participação expressa no engajamento dos filhos com a leitura e desenvolvimento de habilidades leitoras. $O$ desenvolvimento das crianças em leitura mostrou-se motivado pelas mudanças de comportamento dos pais. Isso de deu após a compreensão dos pais sobre a importância e a influência que eles exercem ao fornecer o suporte não apenas material, por meio de livros impressos, mas especialmente na interação leitora com os filhos. No entanto, ficou evidente que mais pesquisas de intervenção nesse sentido devem ser desenvolvidas, em distintos contextos sociais e econômicos, pois é uma prática, até o momento, pouco explorada.

Os estudos que trouxeram aspectos concernentes às diferenças de gênero apontaram dois resultados a serem destacados. Primeiramente, demonstraram que, geralmente, no ambiente familiar há uma tendência maior por parte dos pais para o estímulo da leitura quando se trata de meninas. $O$ outro achado refere-se à tendência de as meninas terem um desempenho melhor em habilidades leitoras e leitura quando comparado com o dos meninos.

O contraste entre países, no que se refere às práticas familiares de leitura, demonstrou que há necessidade de que mais pesquisas sejam feitas em países emergentes e com economia em desenvolvimento. Em países com grande desigualdade sociocultural, o primeiro aspecto que sobressai é o nível socioeconômico que teve a mais forte relação com o desempenho em leitura. Isso demonstra que as crianças provenientes de famílias com níveis socioeconômicos baixos tendem a apresentar escores de leitura também baixos. Além disso, ficou evidente a importância de pesquisas sobre diferentes macrossistemas: além das diferenças econômicas, as diferenças sociais e políticas também podem auxiliar na compreensão de como a organização familiar nos diferentes sistemas impactam a aprendizagem da leitura pelas crianças. 
Entre os estudos revisados, foram poucos os que investigaram os aspectos cognitivos e a hereditariedade dos participantes e suas relações com as diferenças nas habilidades leitoras. Assim, os resultados analisados não dão evidências dessas relações, sugerindo a necessidade de estudos de revisão que se concentrem na busca de pesquisas que investiguem as seguintes variáveis: aspectos cognitivos, hereditariedade e aprendizagem da leitura e compreensão de textos. Entretanto, é possível destacar que, embora fatores genéticos estejam envolvidos na dislexia do desenvolvimento (CARVALHO et al., 2017), o desempenho de crianças sem esse transtorno parece estar muito mais ligado ao contexto de socialização e interação das crianças, com destaque para as práticas familiares de leitura.

Os resultados dos estudos analisados têm importantes implicações educacionais. Eles podem influenciar tanto as políticas públicas voltadas para a educação infantil e os anos iniciais do Ensino Fundamental, como também o desenvolvimento de atividades curriculares que devem ser destacadas nos projetos político-pedagógicos das escolas. No que se refere às políticas públicas, é possível sugerir que sejam feitos mais investimentos na área de educação e cultura, por meio de projetos direcionados para as pessoas de todas as idades, para o desenvolvimento sociocultural da população. Em relação às ações localizadas nas escolas - atividades curriculares - sugere-se que na Educação Infantil e anos iniciais do Ensino Fundamental sejam priorizadas práticas pedagógicas para o desenvolvimento da oralidade, com especial atenção ao desenvolvimento das habilidades metalinguísticas, que são precursoras da aprendizagem inicial e aperfeiçoamento da leitura.

Por último, sugere-se que as iniciativas educacionais já existentes e que venham a ser criadas com o objetivo de atender às necessidades dos estudantes que apresentam poucas oportunidades de leitura e desenvolvimento de habilidades leitoras em casa devem ser acompanhadas e relatadas, de forma a servirem como subsídios de novas intervenções nessa área. 


\section{REFERÊNCIAS}

ARAM, D.; LEVIN, I. Home support of Children in the Writing Process: Contributions to Early Literacy. In: NEUMAN, S. B.; DICKINSON, D. K. Handbook of Early Literacy Research. New York: The Gilford Press, 2011.

BAKER, M.; MILLIGAN, K. Boy-girl differences in parental time investments: Evidence from three countries. National Bureau of Economic Research, Canada, n. 18893, p. 1-50, 2013.

BALTAR, M. A. S.; MOTA, M. M. P. E. Perceived Home Literacy and Reading Performance among Adolescent Brazilian Students. Creative Education, Delaware, v. 7, p. 2836-2844, 2016. DOI: 10.4236/ce.2016.718263.

BENNETT, K. K., WEIGEL, D. J., MARTIN, S. S. Children's acquisition of early literacy skills: examining family contributions. Early Childhood Research Quarterly, Washington, DC. v. 17, n. 3, p. 295-317, 2002.

BERGEN, E. V.; ZUIJEN, T. V.; BISHOP, D.; JONG, P. F. Why Are Home Literacy Environment and Children's Reading Skills Associated? What Parental Skills Reveal. Reading Research Quarterly, Groningen, v. 52, n. 2, p. 147-160, 2016. DOI: 10.1002/rrq.160.

BRITO, P. R.; BROOKS-GUNN, J. Beyond Shared Book Reading: Dimensions of Home Literacy and Low-Income African American Preschoolers' Skills. New Directions for Child and Adolescent Development, San Francisco, v. 92, p. 73-89, 2001.

BURGESS, S. R.; HECHT, S. A.; LONIGAN, C. J. Relationships of the home literacy environment (HLE) to the development of reading-related abilities: A one-year longitudinal study. Reading Research Quarterly, Washington, DC, v. 37, n. 4, p. 408-426, 2002.

CARDOSO, C. V.; MOTA, M. M. P. E. Home-Literacy e os precursores da alfabetização. Estudos e Pesquisas em Psicologia, Rio de Janeiro, v. 15, n. 2, p. 708-724, 2015.

CARVALHO, M. R. S.; SANTOS, F. C.; MARTINS, A. A. S.; BECKER, N.; HAASE, V. G. Fatores genéticos na dislexia do desenvolvimento. In: SALLES, J. F.; NAVAS, A.L. (Orgs.). Dislexias do desenvolvimento e adquiridas. São Paulo: Pearson Clinical Brasil, 2017.

CHIU, M. M.; MCBRIDE-CHANG, C. Family and Reading in 41 Countries: Differences across Cultures and Students. Scientific Studies of Reading, Washington, DC. v. 6, n. 14, p. 514-543, 2010.

COLON, E. G.; ZIMMER-GEMBECK, M. J.; CREED, P. A.; TUCKER, M. Family history, self-perceptions, attitudes and cognitive abilities are associated with early adolescent reading skills. Journal of Research in Reading, Malden, v. 29, n. 1, p. 11-32, 2006.

COMPTON, D. L. The Relationships Among Phonological Processing, Orthographic Processing, And Lexical Development in Children with Reading Disabilities. The Journal of Special Education, North Carolina, v. 35, n. 4, p. 201-210, 2002.

CORREA, J.; MOUSINHO, R. Por um modelo simples de leitura, porém não tão simples assim. In: MOTA, M. P. E.; SPINILLO, A. Compreensão de textos. São Paulo: Casa do Psicólogo, 2013. 
COSTA, K.; MONTIEL, J. M.; BARTHOLOMEU, D.; MURGO, C. S.; CAMPOS, N. R. Percepção do suporte familiar e desempenho em leitura e escrita de crianças do ensino fundamental. Revista Psicopedagogia, São Paulo, v. 33, n. 101, p. 154-163, 2016.

CRUZ, J.; RIBEIRO, I.; VIANA, F. L.; AZEVEDO, H. A leitura de histórias: a qualidade das interações entre pais e filhos. Revista diversidades, Minho, v. 37, p. 16-17, 2012.

DINIZ, E. et al. Influences of developmental contexts and gender differences on School Performance of Children and Adolescents. Educational Psychology, London, v. 34, n. 7, p. 787-798, 2014. DOI: 10.1080/01443410.2013.804491

EDWARDS, C. P. Maternal Literacy Practices and Toddlers' Emergent Literacy Skills. Journal of Early Childhood Literacy, San Francisco, v. 14, n. 1, p. 53-79, 2014. DOI: 10.1177/1468798412451590

ENRICONE, J. R. B.; SALLES, J. R. Relação entre variáveis psicossociais familiares e desempenho em leitura/escrita em crianças. Revista Psicologia Escolar e Educacional, São Paulo, v. 15, n. 2 , p. 199-210, 2011.

ERGÜL, C.; SARICA, A. D.; AKOĞLU, G.; KARAMAN, G. The Home Literacy Environments of Turkish Kindergarteners: Does SES Make a Difference? International Journal of Instruction, Turkey, v. 10, n. 1, p. 187-202, 2017.

EVANS, M.D.; KELLEY, J.; SIKORA, J.; TREIMAN, D.J. Family scholarly culture and educational success: Books and schooling in 27 nations. Research in Social Stratification and Mobility, Nevada, v. 28, n. 2, p. 171-197, 2010. DOI: 10.1016/j.rssm.2010.01.002

GALVÃO, T. F.; PANSANI, T. S. A. Principais itens para relatar Revisões Sistemáticas e Meta-Análises: A recomendação PRISMA. Epidemiol. Serv. Saúde, Brasília, v. 24, n. 2, p. 335-342, 2015.

GUIMARÃES, S. R. K. Dificuldades no Desenvolvimento da Lectoescrita: O Papel das Habilidades Metalinguísticas. Psicologia: Teoria e Pesquisa, Brasília, v. 19, n. 1, p. 33-45, 2003.

GUIMARÃES, S. R. K.; BRANCO, V. Abordagem psicológica da aquisição e desenvolvimento da linguagem escrita: limites e contribuições das teorias. In: RAMOS, E. C.; FRANKLIN, K. (Orgs.). Fundamentos da Educação: os diversos olhares do educar. Curitiba: Juruá, 2010.

HOFF, E. The specificity of environmental influence: Socioeconomic status affects early vocabulary development via maternal speech. Child Development, New York, v. 74, p. 1368-1378, 2003. DOI: $10.1111 / 1467-8624.00612$

JUNG, E. The Development of Reading Skills in Kindergarten Influence of Parental Beliefs About School Readiness, Family Activities, and Children's Attitudes to School. International Journal of Early Childhood, Nederland, v. 48, n. 1, p. 61-78, 2016. DOI 10.1007/s13158-016-0156-2

KAMIL, M.; MOSENTHAL, P. B.; PEARSON, P. D.; BARR, R. Handbook of Reading Research. v. 3, Mahwah, New Jersey: Lawrence Erlbaum Associates, 2000. 
KIM, Y. S. The relationship between home literacy practices and developmental trajectories of emerging literacy and conventional literacy skills for Korean children. Reading and Writing, New York. v. 22, p. 57-84, 2009.

KIRBY, J. R.; HOGAN, B. Family Literacy Environment and Early Literacy Development. Exceptionality Education International, Canada, v. 18, n. 3, p. 112-130, 2008.

KORAT, O.; KLEIN, P.; SEGAL-DRORI, O. Maternal Mediation in Book Reading, Home Literacy Environment, and Children's Emergent Literacy: A Comparison between Two Social Groups. Reading and Writing: An Interdisciplinary Journal, Switzerland, v. 20, n. 4, p. 361-398, 2007.

MAHENDRA, F.; MARIN, A. H. Ambiente familiar e desempenho escolar: Uma revisão sistemática. Psicologia da Educação, São Paulo, v. 40, n. 1, p. 41-57, 2015. DOI: 10.5935/2175-3520.20150004

MATA, L. Literacia Familiar e Desenvolvimento de Competências em Literacia. Exedra Revista Científica, Minho, Número temático, p. 220-227, 2012

MENDIVE, S.; LISSI, M. R.; BAKEMAN, R.; REYES, A. Beyond mother education: maternal practices as predictors of Early Literacy Development in Chilean children from Low-SES Households. Early Education and Development, Washington, DC, v. 28, n. 2, p. 167-181, 2017. DOI: http://dx.doi.org /10.1080/10409289.2016.1197014

NEUMAN, S. B.; DICKINSON, D. K. Handbook of Early Literacy Research. v. 3. New York, NY: The Guilford Press, Editors, 2011. Capítulo 1.

NIKLAS, F.; SCHNEIDER, W. With a Little Help: Improving Kindergarten Children's Vocabulary by Enhancing the Home Literacy Environment. Reading and Writing: An Interdisciplinary Journal, New York, NY, v. 28, n. 4, p. 491-508, 2015.

QUADROS, S.; SARROUB, L. K. The Case of Three Karen Refugee Women and Their Children: Literacy Practices in a Family Literacy Context. Journal of Diaspora, Indigenous, and Minority Education, London, v. 10, n. 1, p. 28-41, 2016.

PURCELL-GATES, V. Family Literacy. In: KAMIL, M.; MOSENTHAL, P. B.; PEARSON, P. D.; BARR, R. Handbook of Reading Research. v. 3. Mahwah, New Jersey: Lawrence Erlbaum Associates, 2000.

PURCELL-GATES, V. Stories, coupons, and the TV-guide: Relationship between home literacy experiences and emergent literacy knowledge. Reading Research Quarterly, Washington, DC. v. 31, p. 406-428, 1996.

RAIKES, H.; PAN; B. A., LUZE, G.; TAMIS-LEMONDA, C. S.; BROOKS-GUNN, J., CONSTANTINE, J. Mother-child book reading in low-income families: Correlates and outcomes during the first three years of life. Child Development, New York, NY, v. 77, n. 4, p. 924-953, 2006. DOI: 10.1111/j.1467-8624.2006.00911.

ROAZZI, A.; HODGES, L.; NOBRE, A. Compreensão de textos e seus processos: para uma didática da compreensão. In: GUIMARÃES, S.R. K.; PAULA, F. V. Compreensão da Leitura: processos cognitivos e estratégias de ensino. São Paulo: Vetor Editora, 2019. 
RODRIGUEZ, E. T.; TAMIS-LEMONDA, C. S.; SPELLMANN, M. E.; PAN, B. A.; RAIKES, H.; LUGO-GIL, J.; LUZE, G. The formative role of home literacy experiences across the first three years of life in children from low-income. Journal of Applied Developmental Psychology, New York, NY. v. 30, p. 677-694, 2009.

SALVADOR, L.; MARTINS, M. A. Práticas de literacia familiar, competências linguísticas e desempenho em leitura no primeiro ano de escolaridade. Análise Psicológica, Lisboa, v. 1, n. 34, p. 1-12, 2017. DOI: $10.14417 / a p .1172$

SANTOS, A. A. A.; JOLY, M. C. R. A. Lendo histórias em família: influências sobre o vocabulário infantil e desempenho em leitura e escrita. Psicologia Escolar e Educacional, Brasília, v. 1, n. 1, p. 39-44, 1996.

SANTOS, P. L.; ALVES, Z. M. M. B. O comportamento de leitura de crianças e adolescentes, segundo a visão das mães. Paidéia, São Paulo, v. 6, p. 62-83, 1994.

SÉNÉCHAL, M.; LEFEVRE, J.; THOMAS, E. M.; DALEY, K. E. Differential Effects of Home Literacy Experiences on the Development of Oral and Written Language. Reading Research Quarterly, New York, NY. v. 33, n. 1, p. 96-116, 1998. DOI: 10.1598/RRQ.33.1.5

SÉNÉCHAL, M.; LEFEVRE, J. Parental involvement in the development of children's reading skill: A five- year longitudinal study. Child Development, New York, NY, v. 73, n. 2, p. 445-460, 2002.

SONNENSCHEIN, S.; MUNSTERMAN, K. The Influence of Home-Based reading interactions on 5-years-olds' reading motivations and early literacy development. Early Childhood Research Quartertly, Washington, DC. v. 17, p. 318-337, 2002.

SPINILLO, A. G. A dimensão social, linguística e cognitiva da compreensão de textos: considerações teóricas e aplicadas. In: MOTA, M. P. E. da; SPINILLO, A. Compreensão de textos. São Paulo: Casa do Psicólogo, 2013.

STORCH, S. A.; WHITEHURST, G. J. The Role of Family and Home in the Literacy Development from Low-Income Backgrounds. New Directions for Child and Adolescent Development, San Francisco, v. 92, p. 53-72, 2001. DOI: 10.1002/cd.15

SWAIN, J.; CARA, O.; MALLOWS, D. We occasionally miss a bath but we never miss stories: Fathers reading to their young children in the home setting. Journal of Early Childhood Literacy, New York, NY. v. 17, n. 2, p. 176-202, 2017.

SWALANDER, L.; TAUBE, K. Influences of Family based prerequisites, Reading attitude, and self-regulation on reading ability. Contemporary Educational Psychology, Belmont, CA. v. 32, p. 206-230, 2007.

UNESCO. United Nations Educational, Scientific and Cultural Organization. Institute for Lifelong Learning. Engaging Families in Literacy and Learning. Hamburg, 2017.

VAN STEENSEL, R. Relations between socio-cultural factors, the home literacy environment and children's literacy development in the first years of primary education. Journal of Research in Reading, New York, NY, v. 29, n. 4, p. 367-382, 2006. 
VASILYEVA, M.; WATERFALL, H. Variability in Language Development: Relation to Socioeconomics Status and Environmental Input. In: NEUMAN, S. B.; DICKINSON, D. K. Handbook of Early Literacy Research. New York, NY: The Guilford Press, 2011.

VIANA, F. L.; RIBEIRO, I.; BARRERA, S. D. Decole - Desenvolvendo competências de letramento emergente: propostas integradoras para a pré-escola. Porto Alegre: Penso, 2017. 